\title{
Epithelial stem cells: the eye provides a vision
}

\begin{abstract}
Epithelial stem cells play a central role in tissue homeostasis, wound repair, and carcinogenesis. Corneal epithelial stem cells have been demonstrated to reside in the limbal epithelium, while the fornical zone of the conjunctiva appears to be a predominant site of conjunctival epithelial stem cells. Stem cells of the corneal and conjunctival epithelia, as well as the hair follicle and interfollicular epidermis share important features: they are capable of self renewal; they are relatively quiescent (slow-cycling); they can be induced to proliferate; and they are multipotent. Its becoming apparent that a certain degree of flexibility exists between corneal and hair follicle keratinocytes.

Eye (2003) 17, 937-942. doi:10.1038/

sj.eye. 6700575
\end{abstract}

Keywords: limbal epithelium; fornical epithelium; bipotent; stem cell flexibility

In its most basic form, the stem cell concept states that within all self-renewing tissues (eg corneal and conjunctival epithelia, epidermis, hair follicle epithelium, intestinal epithelium, and the haematopoietic system) there exists a population of stem cells with a great capacity for self-renewal; when these stem cells divide, they give rise to progeny (transit amplifying or TA cells) that are capable of a finite degree of proliferation. The TA cell, upon exhaustion of its proliferative capacity, becomes postmitotic in the scheme of 'stem cell $\rightarrow$ TA cell $\rightarrow$ postmitotic cell'. Within the TA cell population, a hierarchy exists, ranging from 'young' TA cells capable of many divisions, to more 'mature' TA cells that can divide only a few times. ${ }^{1,2}$ While the stem cell concept, as stated above, has traditionally been viewed as a 'one way street', there is increasing evidence that under certain conditions TA cells can assume a stem cell phenotype (see below).

RM Lavker ${ }^{1}$ and T-T Sun ${ }^{2}$

Slow-cycling: a useful feature for detecting epithelial stem cells

Because of a lack of specific markers for epithelial stem cells, investigators have relied on a combination of cell kinetic, biochemical, and morphological criteria to detect these cells within epithelia. One of the most reliable ways to identify keratinocyte stem cells takes advantage of the fact that these cells are normally slow-cycling, and hence can be identified experimentally as the so-called 'labelretaining cells' (LRCs). ${ }^{2-8}$ The slow-cycling feature is important as it allows stem cells to preserve their proliferative capacity and to minimize DNA replication-associated errors. To detect LRCs, one can label all the cells in the epithelium by a repeated or continuous supply of a deoxynucleoside (eg tritiated thymidine or bromodeoxyuridine - BrdU), followed by a long chase period during which the label is lost from all the cycling TA cells, so that only cells that cycle slowly (the stem cells) retain the label and are designated as LRCs. Using this approach, we and others have showed that the location of LRCs is one of the most reliable criteria to define stem cell-rich regions of various epithelia. For example, we have demonstrated that the corneal epithelium, which had been traditionally regarded as a selfsufficient tissue, contained no LRCs; such cells were found exclusively in the peripheral cornea in the area known as the limbus. ${ }^{2,4}$ Such a result provided strong support to our earlier suggestion that corneal epithelial stem cells reside in the limbus. ${ }^{9}$ By identifying the slowcycling LRCs, we and others have also demonstrated that cells with stem cell characteristics are located at the center of an epidermal proliferative unit ${ }^{6}$ and that, within the hair follicle, they are located in the bulge region of the outer root sheath epithelium..$^{5,8,10,11}$

That these LRCs are indeed stem cells is supported by the fact that they are relatively small and primitive; ${ }^{9}$ they tend to be heavily pigmented in sun-exposed areas and thus are
${ }^{1}$ Department of

Dermatology

The Feinberg School of

Medicine at Northwestern University

Chicago, IL, USA

${ }^{2}$ The Departments of Dermatology, Pharmacology and Urology New York University School of Medicine New York, NY, USA

Correspondence:

RM Lavker

Department of

Dermatology

The Feinberg School of

Medicine at Northwestern University

RM 9-124, 303 E Chicago

Ave Chicago, IL 60611, USA

Tel: +13125034315

E-mail: r-lavker@

northwestern.edu

Received: 28 February 2003 Accepted in revised form: 28 February 2003 
protected from solar damage; ${ }^{12}$ and they have a nonrandom distribution within the epithelium..$^{13,14}$ In addition, they can be induced to proliferate in response to wounding and mitogenic stimuli; ${ }^{2,8}$ they are the targets for neoplastic transformation; ${ }^{15}$ and in some cases have plasticity in that they can form several divergent lineages ${ }^{8,16}$ (see below).

\section{Corneal/limbal epithelium: an ideal system for investigations of stem and TA cells}

The limbal epithelium fulfils more of the criteria associated with epithelial stem cells than any other epithelia where stem cell-enriched regions have been defined.${ }^{14}$ The primary function of the corneal epithelium is to enable light to be transmitted to the interior of the eye, protect the delicate structures of the eye, and to serve as a support for the tear film. The corneal epithelium can be divided into three compartments: limbus, peripheral cornea, and central cornea. The limbal epithelium is an undulating stratified squamous epithelium that overlies a loosely organized stroma containing a network of capillaries. ${ }^{17}$ In contrast, corneal epithelium is a relatively flattened epithelium that overlies a compact 'plywood-like' and avascular stroma. ${ }^{17}$ Specifically, the limbal basal cells are biochemically primitive lacking the corneal epithelial differentiation-associated keratin $\mathrm{K} 3{ }^{9}{ }^{9}$ they contain a subpopulation of cells that rarely cycle and thus can be detected as the label-retaining (slow-cycling) cells; ${ }^{2,4,8}$ they have a high proliferative capacity both in vivo and in vitro ${ }^{17-19}$ they represent the predominant site of corneal tumour formation; ${ }^{20,21}$ they are essential for the long-term maintenance of the corneal epithelium and can be used to reconstitute the entire corneal epithelium in patients with limbal stem cell deficiencies (for review see Tseng and $\operatorname{Sun}^{22}$ ); they give rise to TA cells that undergo centripetal migration; $; 3,24$ they are located in a specialized 'niche' characterized by a loose and wellvascularized stroma; ${ }^{17}$ and they can rescue/reconstitute severely damaged or completely lost corneal epithelium upon transplantation. ${ }^{25-27}$ Conversely, the corneal epithelium lacks label-retaining cells; has a lower proliferative capacity than the limbal epithelium; has a basal cell population that is biochemically more differentiated than the limbal basal cells, and almost never gives rise to tumours (for reviews see Lehrer $e t a l^{2}$ Miller $e t a l_{1}^{13}$ and Pellegrini $\left.e t a l^{18}\right)$. Taken together, these data strongly support, and have led to the wide acceptance of, the concept that corneal epithelial stem cells reside in the limbus ${ }^{28-30}$ and provide a set of welldefined keratinocyte stem cell properties that can aid in the identification of stem cells in other stratified epithelia. ${ }^{14}$ These data also indicate that the corneal epithelial stem cells are well separated from their progeny (TA) cells in the cornea.

\section{Conjunctival epithelium is governed by its own stem cells}

Since the conjunctival epithelium is also a self-renewing tissue, which plays a critical role in maintaining the anterior ocular surface, it is important to consider: (i) is the conjunctival epithelium intrinsically divergent from the corneal epithelium, and thus has its own stem cells; and (ii) if so where are these stem cells located? With regard to the first issue, two lines of evidence demonstrate that this is indeed the case. First, we showed that when various rabbit anterior ocular epithelial cells were grown under identical tissue culture conditions in the presence of a 3T3 feeder layer, limbal and corneal epithelial cells elaborate the corneal-specific K3/K12 keratin pair; however, cultured bulbar, fornical, and palpebral conjunctival epithelial cells, fail to elaborate these corneal keratins. ${ }^{17}$ This suggests that the corneal/ limbal epithelium is intrinsically divergent from the three conjunctival epithelia. A second approach used to test the idea of distinct lineages involves injecting subcutaneously into athymic mice the cultured corneal and conjunctival epithelial cells, and analysing the phenotype of the subcutaneous epithelial cysts. ${ }^{31,32}$ Using this strategy, we found that cultured corneal epithelial cells can reconstitute an epithelial cyst with a corneal epithelial phenotype (formation of a stratified epithelium consisting of basal, wing, and superficial cells), whereas the cultured conjunctival cells reconstitute a conjunctival epithelium consisting of both epithelial and goblet cells. ${ }^{32}$ These two findings strongly support the idea that corneal and conjunctival epithelia represent distinct lineages that are governed by their own stem cells.

Our studies on the cell kinetic and in vitro growth characteristics of the mouse and rabbit bulbar, fornical, and palpebral conjunctival epithelia suggested that the fornical epithelium was enriched in conjunctival epithelial stem cells ${ }^{7,17}$ because: (i) the slow-cycling cells (LRCs) were concentrated in the fornical zone of mouse conjunctiva; 7 (ii) mouse fornical epithelial cells rarely incorporated pulse-administered ${ }^{3} \mathrm{H}-\mathrm{Tdr}$ and/or BrdU;, ${ }^{719}$ (iii) rabbit fornical epithelial cells had the highest in vitro proliferative capacity; ${ }^{17}$ and (iv) following chronic exposure to phorbol myristate, cell proliferation in the fornical epithelium was greater than in the bulbar and palpebral epithelia. ${ }^{19}$ Taken together, these findings led us to suggest that in mice and rabbits, the fornical epithelium is the predominant site of conjunctival epithelial stem cells. ${ }^{7,17,19}$ There could be some species differences in the precise location of conjunctival stem cells. It was recently reported that human bulbar and 
fornical conjunctival cells have an equivalent in vitro proliferative capacity suggesting that human conjunctival epithelial stem cells might be evenly distributed within these two zones. ${ }^{18}$ In addition, it has been suggested that the mucocutaneous junctional epithelium of the rabbit eyelid may be another source of stem cells that helps maintain the palpebral conjunctival epithelium. ${ }^{33}$ Thus the precise location of conjunctival epithelial stem cells is still uncertain and additional investigations are needed to study this important problem.

\section{How epithelia respond to perturbation: lessons from the cornea}

In the corneal/limbal epithelium, stem and TA populations are compartmentalized and can be millimetres apart. This physical separation has allowed us to characterize epithelial stem cells and their progeny TA cell populations, and to gain insight into the proliferative strategies of epithelial wound repair. ${ }^{2}$ Using double-labelling techniques that permit the detection of two or more rounds of DNA synthesis in a given cell, we have investigated the in vivo growth dynamics of stem and TA cell populations in normal and perturbed corneal epithelium. We demonstrated that a large number of normally slow-cycling limbal stem cells could be induced to replicate in response to a single topical application of TPA or to the physical wounding of the central corneal epithelium. In addition, we showed that corneal epithelial TA cells, located in unperturbed peripheral cornea, replicate on average twice and have a relatively long cell cycle time of about $72 \mathrm{~h}$. However, when these same cells were induced to proliferate they greatly shorten their cell cycle time to $\sim 24 \mathrm{~h}$, and undergo additional cell divisions before they become terminally differentiated. Interestingly, central corneal epithelial TA cells, which by definition represent 'older' TA cells than the peripheral TA cells, are able to divide only once prior to becoming postmitotic, both in normal and TPAstimulated corneal epithelium. These observations indicate that a hierarchy exists where young TA cells with relatively high proliferative potential are located in the peripheral corneal epithelium, while the more mature TA cells having relatively little proliferative reserve are located in the central corneal epithelium (Figure 1). These results thus demonstrated three strategies that corneal epithelium can employ to expand its cell population during wound healing: (i) recruitment of the normally slow-cycling stem cells to divide, thus producing more TA cells; (ii) increasing the number of times a TA cell can replicate before it becomes postmitotic; and (iii) increasing the efficiency of TA cell replication by shortening the cell cycle time. ${ }^{2}$

\section{How flexible are the epithelial stem cells?}

One of the most exciting areas of stem cell biology stems from the recent demonstration of the interconvertability of haematopoietic cells and neuronal tissues, ${ }^{34,35}$ skeletal muscle cells, ${ }^{36}$ and hepatic stem cells. ${ }^{37,38}$ This raises the question of whether corneal epithelial stem cells are also plastic. It has been demonstrated in several epithelia that a single progenitor cell (stem cell) can give rise to phenotypically distinct cell populations. For example, single clones of conjunctival epithelial cells can give rise to epithelial cells and goblet cells; ${ }^{39}$ the intestinal stem cell gives rise to enterocytes, goblet cells, paneth cells, and enteroendocrine cells; ${ }^{40}$ and the airway epithelial

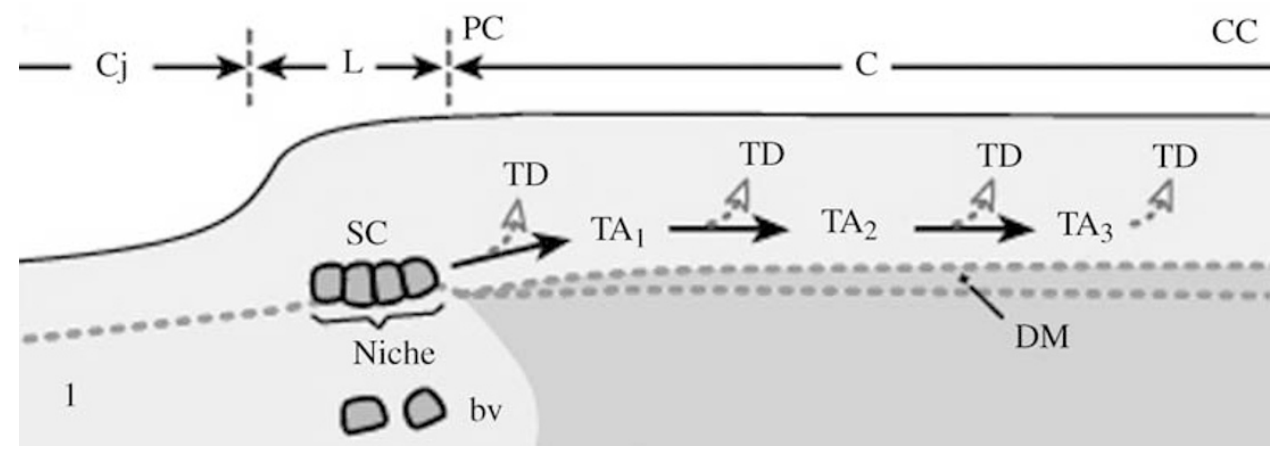

Figure 1 Schematic diagram showing the location of corneal epithelial stem cells (SC) in the basal layer of the limbus. These stem cells overlie a mesenchyme (niche) that is distinctively more cellular and blood vessel (bv) rich than the corneal stroma. Solid arrows denote the centripetal ('horizontal') migration of limbal-derived TA cells which progressively lose their proliferative potential; dashed arrows denote the ('vertical') migration of cells into the suprabasal compartment to become terminally differentiated (TD). Other abbreviations: C (cornea), CC (central cornea), Cj (conjunctiva), DM (Descemet membrane), L (limbus), and PC (peripheral cornea). ${ }^{8}$ 


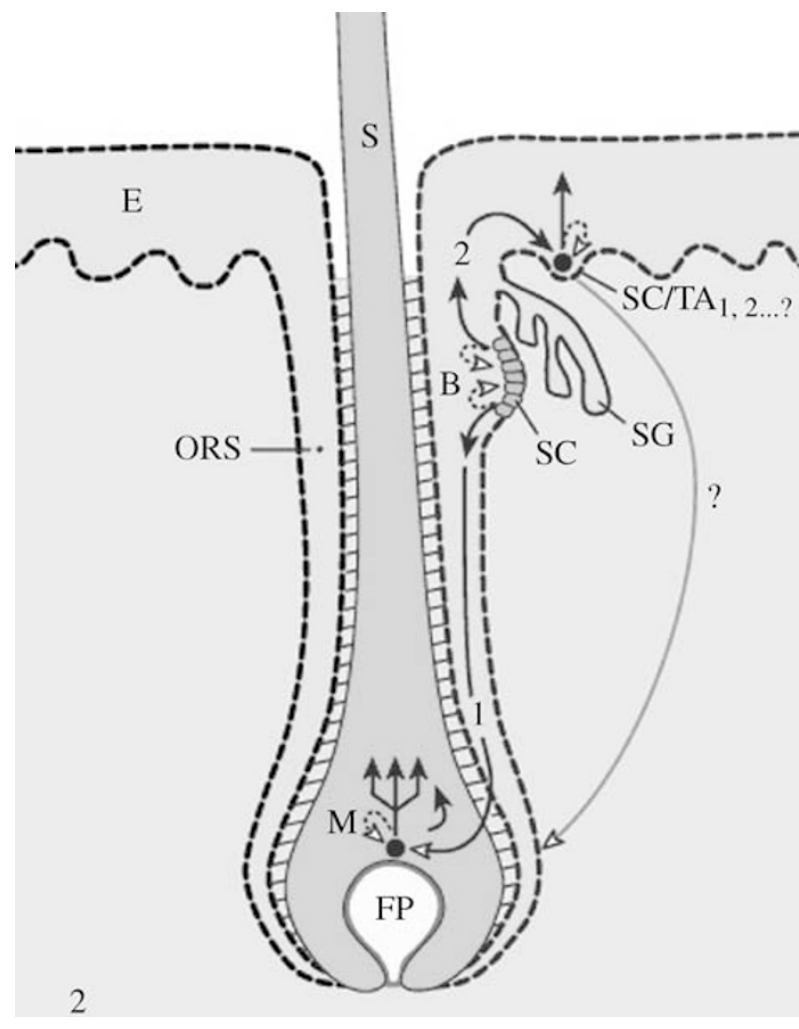

Figure 2 Schematic diagram of an epidermo-pilosebaceous unit in hair-bearing skin. The unit consists of the epidermis and the hair follicle with its associated sebaceous gland. The bulge contains a population of putative keratinocyte stem cells that can give rise to (pathway 1) a population of pluripotent and rapidly dividing progenitor (transit amplifying) cells in the matrix that yields the hair shaft. Alternatively, the bulge stem cells can give rise to the stem/progenitor cells of the epidermis (pathway 2). It is hypothesized here that the epidermal stem cell represents a form of bulge-derived, young transit amplifying cell (SC/ $\mathrm{TA}_{1,2} \ldots$ ?). The long, curved arrow denotes the demonstrated capability of adult epidermal cells to form a new hair follicle in response to appropriate mesenchymal stimuli. Abbreviations: $\mathrm{B}$ (bulge), E (epidermis), FP (follicular or dermal papilla), M (matrix keratinocytes), ORS (outer root sheath), S (hair shaft), SC (stem cells), and TA (transit amplifying cells). ${ }^{14}$

stem cell can give rise to support cells, goblet cells, and clara cells. ${ }^{41-43}$ A recent example of the multipotency of epithelial stem cells is provided by hair follicle epithelial stem cells. Using a double-labelling method that selectively tagged the progeny (TA) cells of the bulge stem cells, we showed that these bulge-derived TA cells can move not only downward to form the outer root sheath, matrix, and the hair shaft of the hair follicle, but also upward to populate the epidermal basal layer. ${ }^{8}$ The emigration of bulge-derived follicular TA cells into the epidermis was observed in normal neonatal mouse skin, as well as in adult skin in response to a full-thickness skin wound. Such an upward emigration of follicular epithelial TA cells into even normal epidermis suggests a much closer relation between the follicle and normal epidermis than was previously appreciated, and supports the idea that bulge-derived keratinocytes may be involved in the long-term maintenance of the epidermis..$^{5,10,44}$ The fact that the bulge stem cells are involved in the formation of the hair follicle as well as the epidermis suggests that the bulge cells are at least bipotent (Figure 2). ${ }^{8,10,14}$ Microdissection studies by Oshima et $a l^{16}$ demonstrated that the $\beta$-galactosidasetagged bulge cells of mouse vibrissae follicles can give rise to not only the entire follicle but also the epidermis. The plasticity of corneal epithelial stem cells and their progeny was recently demonstrated by Ferraris et al ${ }^{45}$ who studied tissue recombinants, consisting of rabbit central corneal epithelium (comprised of TA cells) and embryonic mouse dermis, which were grafted onto nude mice and followed temporally. They found that corneal epithelial cells gave rise to hair follicle buds, which matured into fully formed hair follicles, and eventually the overlying corneal epithelium assumed an epidermal phenotype including the expression of the epidermal K10 keratin rather than the $\mathrm{K} 3 / \mathrm{K} 12$ corneal keratin pair. ${ }^{45}$ These findings indicate that, given the appropriate signal(s), the TA cells of central corneal epithelium can be converted to become epidermis and its appendages, thus proving that even the relatively mature TA cells of central cornea can retain significant developmental flexibility.

In conclusion, available data strongly support the ideas that: (i) corneal epithelial stem cells reside in the limbal epithelium; (ii) corneal and conjunctival epithelia are intrinsically divergent and are governed by separate epithelial stem cells; (iii) rodent conjunctival epithelial stem cells reside in the fornical zone of the conjunctival epithelium; (iv) conjunctival epithelial stem cells are bipotent; and (v) there appears to be flexibility between corneal and hair follicle keratinocytes. What is needed is a better understanding of the signals that cause the normally quiescent stem cells to divide and to give rise to young multipotent TA cells, and of the degree of plasticity that exists within the keratinocyte stem cell and transit amplifying cell compartments.

\section{Acknowledgements}

This work was supported by National Institutes of Health Grants EY06769, EY13711 (RML) and DK39753, DK52206, DK52643, DK57629 (T-TS).

\section{References}

1 Loeffler M, Potten CS, Wichmann HE. Epidermal cell proliferation. II. A comprehensive mathematical model of cell proliferation and migration in the basal layer predicts 
some unusual properties of epidermal stem cells. Vichows Arch B 1987; 83: 286-300.

2 Lehrer MS, Sun T-T, Lavker RM. Strategies of epithelial repair: modulation of stem cell and transit amplifying cell proliferation. J Cell Sci 1998; 111: 2867-2875.

3 Bickenbach JR. Identification and behavior of label-retaining cells in oral mucosa and skin. J Dent Res 1981; 60: 1611-1620.

4 Cotsarelis G, Cheng SZ, Dong G, Sun T-T, Lavker RM. Existence of slow-cycling limbal epithelial basal cells that can be preferentially stimulated to proliferate: implications on epithelial stem cells. Cell 1989; 57: 201-209.

5 Cotsarelis G, Sun T-T, Lavker RM. Label-retaining cells reside in the bulge of the pilosebaceous unit: implications for follicular stem cells, hair cycle, and skin carcinogenesis. Cell 1990; 61: 1329-1337.

6 Morris RJ, Potten CS. Slowly cycling (label-retaining) epidermal cells behave like clonogenic stem cells in vivo. Cell Prolif 1994; 27: 279-289.

7 Wei ZG, Cotsarelis G, Sun T-T, Lavker RM. Label-retaining cells are preferentially located in fornical epithelium: implications on conjunctival epithelial homeostasis. Invest Ophthalmol Vis Sci 1995; 36: 236-246.

8 Taylor G, Lehrer MS, Jensen PJ, Sun T-T, Lavker RM. Involvement of follicular stem cells in forming not only the follicle but also the epidermis. Cell 2000; 102: 451-461.

9 Schermer A, Galvin S, Sun T-T. Differentiation-related expression of a major $64 \mathrm{~K}$ corneal keratin in vivo and in culture suggests limbal location of corneal epithelial stem cells. J Cell Biol 1986; 103: 49-62.

10 Lavker RM, Miller S, Wilson C, Cotsarelis G, Wei ZG, Yang JS et al. Hair follicle stem cells: their location, role in hair cycle, and involvement in skin tumor formation. J Invest Dermatol 1993; 101: 16s-26s.

11 Morris RJ, Potten CS. Highly persistent label-retaining cells in the hair follicles of mice and their fate following induction of anagen. J Invest Dermatol 1999; 112: 470-475.

12 Davanger M, Evensen A. Role of the pericorneal papillary structure in renewal of corneal epithelium. Nature 1971; 229: 560-561.

13 Miller SJ, Lavker RM, Sun T-T. Keratinocyte stem cells of cornea, skin and hair follicle: common and distinguishing features. Semin Dev Biol 1993; 4: 217-240.

14 Lavker RM, Sun T-T. Epidermal stem cells: properties, markers and location. Proc Natl Acad Sci USA 2000; 97: 13473-13475.

15 Morris RJ, Coulter K, Tryson K, Steinberg SR. Evidence that cutaneous carcinogen-initiated epithelial cells from mice are quiescent rather than actively cycling. Cancer Res 1997; 57: 3436-3443.

16 Oshima H, Rochat A, Kedzig C, Kobayashi K, Barrandon Y. Morphogenesis and renewal of hair follicles from adult multipotent stem cells. Cell 2001; 104: 233-245.

17 Wei ZG, Wu RL, Lavker RM, Sun T-T. In vitro growth and differentiation of rabbit bulbar, fornix, and palpebral conjunctival epithelia. Implications on conjunctival epithelial transdifferentiation and stem cells. Invest Ophthalmol Vis Sci 1993; 34: 1814-1828.

18 Pellegrini G, Golisano O, Paterna P, Lambiase A, Bonini S, Rama $\mathrm{P}$ et al. Location and clonal analysis of stem cells and their differentiated progeny in the human ocular surface. J Cell Biol 1999; 145: 769-782.

19 Lavker RM, Wei Z-G, Sun T-T. Phorbol ester preferentially stimulates mouse fornical conjunctival and limbal epithelial cells to proliferate in vivo. Invest Ophthalmol Vis Sci 1998; 39: 301-307.

20 Pizzarello LD, Jakobiec FA. Bowen's disease of the conjunctiva. In: Jakobiec FA (ed). Ocular and Adenexal Tumors. Aesculappius: Birmingham; 1978, pp 553-571.

21 Russell WO, Wynne ES, Loquvam GS. Studies on bovine ocular squamous carcinoma ('cancer eye'). Cancer 1956; 9: $1-52$.

22 Tseng SCG, Sun T-T. Stem cells: ocular surface maintenance. In: Brightbill FS (ed). Corneal Surgery: Theory, Technique, Tissue. Mosby: St Louis, MO, 1999, pp 9-18.

23 Buck RC. Measurement of centripetal migration of normal corneal epithelial cells in the mouse. Invest Ophthalmol Vis Sci 1985; 26: 1296-1299.

24 Auran JD, Koester CJ, Kleiman NJ, Rapaport R, Bomann JS, Wirotsko BM et al. Scanning slit confocal microscopic observations of cell morphology and movement within the normal human anterior cornea. Ophthalmology 1995; 102: 33-41.

25 Tsubota K, Toda I, Saito H, Shinozaki N, Shimazaki J. Reconstruction of the corneal epithelium by limbal autograft transplantation for corneal surface reconstruction. Ophthalmology 1995; 102: 1486-1496.

26 Pelligrini G, Traverse CE, Franzi TT, Zingirrau M, Cacedda $\mathrm{R}$, DeLuca M. Long-term restoration of damaged corneal surfaces with autologous cultivated corneal epithelium. Lancet 1997; 349: 990-993.

27 Tsai RJF, Li L-M, Chen J-K. Reconstruction of damaged corneas by transplantation of autologous limbal epithelial cells. N Engl J Med 2000; 343: 86-93.

28 Dua HS. Stem cells of the ocular surface: scientific principles and clinical applications. Br J Ophthalmol 1995; 79: 968-969.

29 Hodson S. Cultivating a cure for blindness. Nature 1997; 387: 449 .

30 Voelker R. Stem cells hold vision for the future. JAMA 1997; 278: 1477-1478.

31 Doran TI, Vidrich A, Sun TT. Intrinsic and extrinsic regulation of the differentiation of skin, corneal and esophageal epithelial cells. Cell 1980; 22: 17-25.

32 Wei Z-G, Sun T-T, Lavker RM. Rabbit conjunctival and corneal epithelial cells belong to two separate lineages. Invest Ophthalmol Vis Sci 1996; 37: 523-533.

33 Wirthschafter JD, Ketcham JM, Weinstock RJ, Tabesh T, McLoon LK. Mucocutaneous junction as the major source of replacement palpebral conjunctival epithelial cells. Invest Ophthalmol Vis Sci 1999; 40: 3138-3146.

34 Bjornson CR, Rietze RL, Reynolds BA, Magli MC, Vescovi AL. Turning brain into blood: a hematopoietic fate adopted by adult neural stem cells in vivo. Science 1999; 283: 534-537.

35 Mezey E, Chandross KJ, Harta G, Maki RA, McKercher SR. Turning blood into brain: cells bearing neuronal antigens generated in vivo from bone marrow. Science 2000; 290: 1779-1782.

36 Jackson KA, Mi T, Goodell MA. Hematopoietic potential of stem cells isolated from murine skeletal muscle. Proc Natl Acad Sci USA 1999; 96: 14482-14486.

37 Petersen BE, Bowen WC, Patrene KD, Mars WM, Sullivan AK, Murase $\mathrm{N}$ et al. Bone marrow as a potential source of hepatic oval cells. Science 1999; 284: 1168-1170.

38 Lagasse E, Connors H, Al-Dhalimy M, Reitsma M, Dohse $\mathrm{M}$, Osborne L et al. Purified hematopoietic stem cells can differentiate into hepatocytes in vivo. Nat Med 2000; 6: 1229-1234. 
39 Wei Z-G, Sun T-T, Lavker RM. Keratinocyte and goblet cells of the rabbit conjunctival epithelium share a common progenitor cell. Invest Ophthalmol Vis Sci 1997; 36: 422s.

40 Potten CS, Booth C, Pritchard DM. The intestinal epithelial stem cell: the mucosal governor. Int J Exp Pathol 1997; 78: 219-243.

41 Johnson NF, Hubbs AF. Epithelial progenitor cells in the rat trachea. Am J Respir Cell Mol Biol 1990; 3: 579-585.

42 Zepeda ML, Chinoy MR, Wilson JM. Characterization of stem cells in human airway capable of reconstituting a fully differentiated bronchial epithelium. Somat Cell Mol Genet 1995; 21: 61-73.
43 Borthwick DW, Shahbazian M, Krantz QT, Dorin JR, Randell $\mathrm{SH}$. Evidence for stem-cell niches in the tracheal epithelium. Am J Respir Cell Mol 2001; 24: 662-670.

44 Yang JS, Lavker RM, Sun TT. Upper human hair follicle contains a subpopulation of keratinocytes with superior in vitro proliferative potential. J Invest Dermatol 1993; 101: 652-659.

45 Ferraris C, Chevalier G, Favier B, Jahoda CA, Dhouailly D. Adult corneal epithelium basal cells possess the capacity to activate epidermal, pilosebaceous and sweat gland genetic programs in response to embryonic dermal stimuli. Development 2000; 127: 5487-5495. 